\title{
PsvWave: Elastic Wave Propagation in 2D for Python and C ++
}

\author{
Lars Gebraad (D) $1^{*}$ and Andreas Fichtner (D) 1 \\ ${ }^{1}$ Department of Earth Sciences, ETH Zürich
}

Non-PEER REVIEWEd PREPrint SUbMitTed to EARThArXIV, COMPILEd FEbruARY 22, 2022

\begin{abstract}
We present 'psvWave', a basic numerical finite difference solver for Python and C++, specifically targeted at seismologists. The solver is based on the well-established staggered grid approaches developed for the P-SV elastic wave equation. Although its functionality is limited (solely moment tensor sources, only Ricker wavelets source time functions), it does possess the ability to perform adjoint simulations, and its performance has so far allowed the development of Bayesian sampling for Full-Waveform Inversion using the Hamiltonian Monte Carlo algorithm. We present this as an open source project, and invite anyone to contribute.
\end{abstract}

\section{INTRODUCTION}

This software was born out of the need to simulate many small wavefields quickly, from a Python environment. As such, we implemented Virieux's [1] seminal work in a C++ OpenMP enabled code that interfaces with Python. Configuration and functionality is minimal, and therefore so is overhead. Its fast performance has so far allowed it to be used for Hamiltonian Monte Carlo sampling with hundreds of thousands of simulations performed in a short time [2]. The software can perform 'forward' as well as 'adjoint' computations, thereby facilitating the computation of sensitivity kernels relevant to various inverse theory methods [3]. It should be noted that the main aim of psvWave is research, and we do not consider it suited for production.

\section{The solver and Python InTerface}

The psvWave package contains a forward and an adjoint $2 \mathrm{~d}$ elastic wave equation solver. The staggered grid as well as the leap-frog time integration are equal to that described in [1] Additionally, the $\mathrm{C}++$ core allows one to compute misfits w.r.t some observed data, and to subsequently calculate sensitivity kernels using the resulting adjoint sources. The Python interface gives access to all core functionality, but also extends the $\mathrm{C}++$ functionality by providing plotting functions.

The simulations performed make a few basic assumptions about the medium, wavefield and sources, as given below.

\subsection{Assumptions}

All sources propagate waves through the same medium / domain in the $x, z$-plane, and are recorded by the same network of receivers. The physics are for in-plane shear waves and defined in a right-handed coordinate system. However, one can interpret the simulations in any unit and orientation. One should make sure that all units used result in wavefields that are within the range of $\mathrm{C}++$ doubles.

All sources are normal / reverse faults (with strike parallel to the y-axis) using a Ricker wavelet as source time function. Every source can have a different dip angle. This source time function can be altered in both the Python and $\mathrm{C}++\mathrm{API}$, the focal mechanism / source type not.
Simulations are divided in 'shots', i.e. a single time length in which data is recorded and some sources 'fire'. The code allows for time staggering of sources, i.e. 'firing' multiple sources in a single simulation.

The domain is truncated on all 4 sides by absorbing boundary conditions. It's width is variable, but as of yet, the same on all sides. This does not directly allow for free boundary conditions, but this is planned to change. When measuring distance or counting gridpoints, the zero-point is the first points not inside the boundary layer but in the actual simulation medium. When updating medium properties within the domain, the boundary copies the medium properties closest to it, to avoid creating reflectors.

The location of the sources and receivers is not expressed in distance, but in gridpoint numbering. Because the actual indexing starts within the medium, and not the absorbing boundary, sources and receivers can only be placed inside the medium. However, the and variables determine how many gridpoints are not considered free parameters. The idea behind this is that this allows us to place sources/receivers in regions of the domain that are not inverted for, and are also not inside the boundary. This to avoid near-source and near-receiver effects.

\subsection{Known limitations}

The solver does not employ checkpoint, but simply stores the dynamical fields (particle motion, strain) at specific intervals. This setting, found in the configuration files as inversion.snapshot_interval allows one to reduce the storage in host machines RAM, but also deteriorates the precision of the computed sensitivity kernels if it is chosen too high.

Additionally, all simulations store their wavefields separately. By growing the number of simulations in a single modeller (by e.g. adding more sources), the memory required to store the wavefields (for subsequent adjoint simulations) grows linearly. This could be circumvented by running all forward and adjoint simulations sequentially and re-using the memory used between sources, but is as of yet not implemented.

The misfit that is implemented in the package, with its adjoint source, is currently only the L2 misfit. The documentation illustrated how one can smooth the gradients for this misfit. 


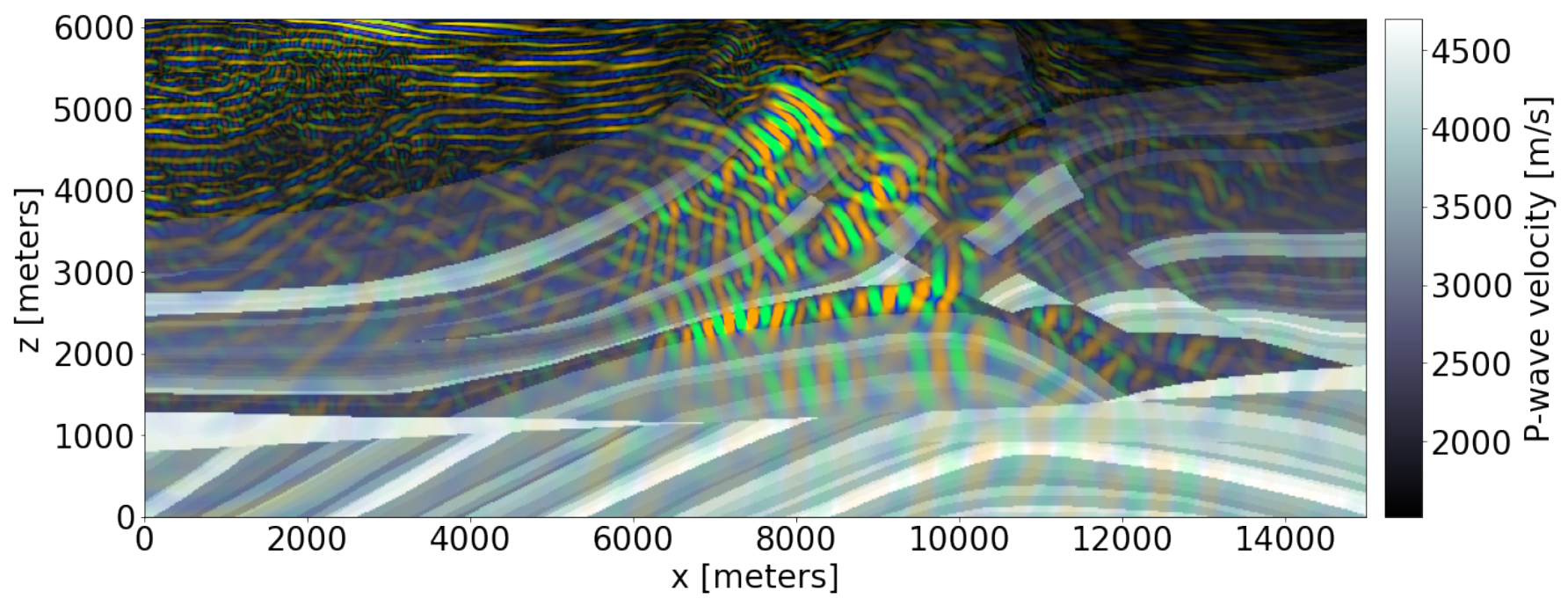

Figure 1: Horizontal (yellow-blue) and vertical (orange-green) particle motion from Notebook 1 superimposed on the P-wave velocity model of the elastic Marmousi model.

\section{AVAILABILITY, INSTRUCTIONS AND TUTORIALS}

In the project repository [4], we provide installation instructions for PyPi installation, as well as a dedicated Docker image. The PyPi installation only supports Linux AMD64 architectures. The Docker is fully multiplatform and requires no set-up. Additionally, the repository provides two notebooks. Notebook 1 concerns itself with the configuration file and performing forward simulations, as well as the visualization of the outputs. Notebook 2 demonstrates how psvWave combined with the L-BFGS [5] algorithm allows one to perform deterministic full-waveform inversion.

\section{AcKNOWLEDGEMENTS}

We would like to thank all the open source software projects that allow psvWave to function. Specifically, we make heavy use of Eigen [6], PyBind11 [7], NumPy [8], SciPy [9] and Matplotlib [10]. Our code runs on any platform thanks to Docker. This document is a non-peer reviewed work published on EarthArXiv.org.

\section{REFERENCES}

[1] Jean Virieux. P-sv wave propagation in heterogeneous media: Velocity-stress finite-difference method. Geophysics, 51(4):889-901, 1986.

[2] Lars Gebraad, Christian Boehm, and Andreas Fichtner. Bayesian Elastic Full-Waveform Inversion Using Hamiltonian Monte Carlo. Journal of Geophysical Research: Solid Earth, 125(3):e2019JB018428, 2020. ISSN 2169-9356. doi: 10.1029/2019JB018428.

[3] Andreas Fichtner, H-P Bunge, and Heiner Igel. The adjoint method in seismology: I. theory. Physics of the Earth and Planetary Interiors, 157(1-2):86-104, 2006.

[4] Lars Gebraad and Andreas Fichtner. psvWave, 22022. URL https://github.com/larsgeb/psvWave.
[5] Jorge Nocedal. Updating quasi-newton matrices with limited storage. Mathematics of computation, 35(151):773782,1980 .

[6] Gaël Guennebaud, Benoît Jacob, et al. Eigen v3. http://eigen.tuxfamily.org, 2010.

[7] Wenzel Jakob, Jason Rhinelander, and Dean Moldovan. pybind11 - seamless operability between $\mathrm{c}++11$ and python, 2017. https://github.com/pybind/pybind11.

[8] Charles R. Harris, K. Jarrod Millman, Stéfan J. van der Walt, Ralf Gommers, Pauli Virtanen, David Cournapeau, Eric Wieser, Julian Taylor, Sebastian Berg, Nathaniel J. Smith, Robert Kern, Matti Picus, Stephan Hoyer, Marten H. van Kerkwijk, Matthew Brett, Allan Haldane, Jaime Fernández del Río, Mark Wiebe, Pearu Peterson, Pierre Gérard-Marchant, Kevin Sheppard, Tyler Reddy, Warren Weckesser, Hameer Abbasi, Christoph Gohlke, and Travis E. Oliphant. Array programming with NumPy. $\mathrm{Na}$ ture, 585(7825):357-362, September 2020. doi: 10.1038/ s41586-020-2649-2.

[9] Pauli Virtanen, Ralf Gommers, Travis E. Oliphant, Matt Haberland, Tyler Reddy, David Cournapeau, Evgeni Burovski, Pearu Peterson, Warren Weckesser, Jonathan Bright, Stéfan J. van der Walt, Matthew Brett, Joshua Wilson, K. Jarrod Millman, Nikolay Mayorov, Andrew R. J. Nelson, Eric Jones, Robert Kern, Eric Larson, C J Carey, İlhan Polat, Yu Feng, Eric W. Moore, Jake VanderPlas, Denis Laxalde, Josef Perktold, Robert Cimrman, Ian Henriksen, E. A. Quintero, Charles R. Harris, Anne M. Archibald, Antônio H. Ribeiro, Fabian Pedregosa, Paul van Mulbregt, and SciPy 1.0 Contributors. SciPy 1.0: Fundamental Algorithms for Scientific Computing in Python. Nature Methods, 17:261-272, 2020. doi: 10.1038/s41592-019-0686-2.

[10] J. D. Hunter. Matplotlib: A 2d graphics environment. Computing in Science E Engineering, 9(3):90-95, 2007. doi: 10.1109/MCSE.2007.55. 